of hyperekplexia to chromosome $5 \mathrm{q}$. The hypothesis that a mutation in a GABA-receptor subunit causes hyperekplexia is particularly attractive in view of the long term benzodiazepine responsiveness of the disorder."

\title{
EYE MOVEMENT DISORDERS
}

\section{PERIODIC ALTERNATING GAZE AND CEREBELLAR DYSGENESIS}

Two children with periodic alternating gaze deviation, presenting at birth in 1 patient and at 2 months in the other, are reported from the New England Medical Center, Tufts University, Boston, MA. The eyes conjugately drifted from one side to the other. With the head stabilized, a regular nystagmus of low frequency and large amplitude was seen when the patient attempted to direct the eyes away from the gaze bias. Compensatory head turning was noted in both patients. MRI showed multiple congenital abnormalities of the posterior cranial fossa. In 1 patient a left renal aplasia and a right supra-adrenal neuroblastoma were diagnosed by ultrasonography and by resection at 4 weeks of age. (Legge $\mathrm{RH}$ et al. Periodic alternating gaze deviation in infancy. Neurology Sept 1992; 42:1740-1743.) (Reprints: Dr. Thomas R. Hedges III, 750 Washington Street, Box 381, Boston, MA 02111.)

COMMENT. The periodic alternating gaze phenomenon is rare and points to cerebellar disease, with malformation of the inferior cerebellar vermis as the specific underlying lesion. The association with neuroblastoma in 1 patient is a coincidence since symptoms of opsoclonus were not described.

\section{OCULOMOTOR PARESIS AND BASILAR ANEURYSM}

The clinical and pathologic findings in a 10 month old girl with congenital heart diseasewho died after rupture of a congenital distal basilar artery aneurysm are reported from the University of Connecticut Health Center, Farmington, CT and the Children's Hospital of Philadelphia, PA. She was hospitalized at 10 months of age after having had 4 syncopal episodes within a 6 week interval. Twenty-four hours after cardiac catherization the infant developed an adduction deficit of the left eye which resolved over the next 36 hours. Five days after surgical correction of the cardiac defect she had a massive subarachnoid hemorrhage. (DiMario FJ Jr., Rorke LB. Transient oculomotor nerve paresis in congenital distal basilar artery aneurysm. Pediatr Neurol July/Aug 1992; 8:303-306.) (Communications: Dr. DiMario, Division of Neurology, University of Connecticut Health Center, 263 Farmington Avenue, Farmington, CT 06030.)

COMMENT. Transient third nerve paresis in an infant with a history of recurrent syncope and congenital heart disease should suggest a possible intracranial arterial aneurysm. The authors found 6 previous reports of basilar aneurysm preceded by a transient third nerve dysfunction. A migraine variant is another explanation. (Shevell MI

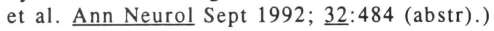

